\title{
Experimental and numerical studies on the braiding of carbon fibres over structured end-fittings for the design and manufacture of high performance hybrid shafts
}

\author{
Xiaochuan Sun ${ }^{1} \cdot$ Luiz F. Kawashita ${ }^{1}$ (D) Tino Wollmann ${ }^{2} \cdot$ Sebastian Spitzer² ${ }^{2}$ Albert Langkamp ${ }^{2} \cdot$ Maik Gude $^{2}$
}

Received: 13 December 2017 / Accepted: 21 March 2018 / Published online: 26 April 2018

(c) The Author(s) 2018

\begin{abstract}
Braiding is an attractive manufacturing method for tubular elements such as hollow shafts and struts. One of the main challenges however is the integration of suitably performing end-fittings. Recent advances in additive layer manufacture have enabled the fabrication of end-fittings which can be 'co-impregnated' or 'co-cured' with the fibre preform in a single step, i.e. without the need for secondary adhesive bonding. This requires the introduction of protrusions onto the surface of the end-fitting to promote mechanical interlocking with the fibres. However, the lack of accurate modelling tools for the simulation of this manufacturing process means that much empiricism is currently used in the design of such structures. A novel numerical framework is presented here for the full-scale simulation of the braiding process over structured end-fittings. Nonlinear finite element analysis is applied at the meso-scale, with strands of beam elements representing individual yarns and meshed surfaces modelling the mandrel and tooling. Penalty-based contact formulations are then used to simulate all inter-yarn and yarn-metal interactions, enabling detailed predictions of fibre paths around surface protrusions. In order to verify and validate this numerical framework, a series of full-scale braiding experiments was conducted using additivelymanufactured thermoplastic mandrels. Final braid patterns as well as the occurrence of braid imperfections were investigated and compared to model predictions. It is shown that the proposed modelling strategy reproduces well the trends observed experimentally in terms of final braid quality. A parametric study was then conducted on the effects of initial end-fitting alignment with respect to oncoming yarns, suggesting that better control over this parameter could reduce considerably the occurrence of braid imperfections.
\end{abstract}

Keywords Process modelling · Braiding $\cdot$ Carbon fibre composites $\cdot$ Finite element analysis $\cdot$ Hybrid joints $\cdot$ Additive layer manufacture $\cdot$ Virtual testing

\section{Introduction}

The use of composite materials, such as fibre reinforced polymers (FRPs) has been increasing rapidly in several industries including aerospace, automotive and renewable energy, due to the superior specific strength and stiffness of these materials over their metallic counterparts. However,

Xiaochuan Sun

Ric.Sun@bristol.ac.uk

1 Bristol Composites Institute (ACCIS), University of Bristol, University Walk, BS8 1TR Bristol, UK

2 Technische Universität Dresden, Institute of Lightweight Engineering and Polymer Technology, Holbeinstraße 3, 01307 Dresden, Germany because of their limited operation temperatures, poor wear resistance and relatively low through-thickness strength, composites are often used in combination with metals in primary structural components. The performance of such hybrid structures can be enhanced by the use of metal-composite 'hybrid joints' -i.e. those combining adhesion and mechanical interlocking. This is particularly the case for the manufacture of lightweight struts and shafts in aerospace applications, and more recently also in high-end automotive applications.

In order to enhance the through-thickness properties of FRPs and the interfacial properties between metal and composite, 'structured' surfaces may be used, i.e. those containing patterns of relatively small protrusions which do not incur significant weight penalty. The design, manufacture and optimisation of hybrid joints with structured metal 
surfaces has become the focus of much research in recent years. This is mainly attributed to the rapid development of advanced metal manufacturing techniques such as cold metal transfer, electron beam machining, laser beam welding, and more recently additive layer manufacture (ALM), in particular selective laser sintering of metal powders.

Cartié et al. [1] demonstrated how FRP and metallic pins can be used to reinforce laminated composites in the out-ofplane direction through 'crack bridging' mechanisms, drastically improving the effective interlaminar toughness of such materials. Ucsnik et al. [2, 3] investigated the mechanical properties of hybrid metal-composite joints where surface pins were introduced via cold metal transfer, resulting in drastic improvements in tensile strength, failure strain and total energy absorption. Graham et al. [4] trialled several configurations of multi-material hybrid joints produced mainly via laser metal deposition and cold metal transfer. Wang et al. [5] studied hybrid joints between carbon fibre reinforced polymers (CFRP) and titanium Ti-6Al-4V, using the Comeld ${ }^{\mathrm{TM}}$ electron beam machining technology [6] to sculpt surface protrusions which enhanced bond strength considerably. Working with a similar material combination, Kashaev et al. [7] investigated 'laser rivetted' hybrid joints, observing mechanical properties which were comparable to those of traditional riveted joints. Ko et al. [8] characterised hybrid joints made of stainless steel 'z-pins' under fatigue and environmental conditions, with pins being worked mechanically prior to insertion for increased surface roughness and hence improved crack bridging performance.

Nguyen et al. [9] studied hybrid joints between Ti-Ti and Ti-CFRP substrates, with surface features manufactured via selective laser melting (SLM) of metal powders. They found that macro-scale surface features (e.g. dimples or grooves) combined with the inherent roughness of SLM surfaces, resulted in superior bond fracture toughness. These same authors later characterised the pull-out performance of metal-composite joints with SLM surfaces considering various pin geometries and offset angles [10,11].

Compared to other techniques, ALM offers the greatest freedom of design and homogeneity of material properties, at the expense of high manufacturing costs and some level of porosity in the final product. The rough surface finish obtained from powder-based ALM-which in most applications is unwanted and requires post-processing steps-is advantageous in the case of hybrid joints as it increases the total surface area of the interface, which in turn improves adhesion $[12,13]$.

The references discussed here so far focused on the characterisation of planar joints. Few studies have investigated cylindrical hybrid joints produced via braiding or winding processes. Braiding is a mature technique particularly suitable for the manufacture of high-performance rope-like fibre preforms (2D braiding) or thick-walled preforms (3D braiding) at relatively high production rates. These high rates are possible because large numbers of fibre yarns can be deposited simultaneously, as opposed to the few yarns deposited at once in alternative techniques (e.g. filament winding). In standard 2D braiding, yarns are drawn from bobbins attached to carriers which rotate around a stationary circular frame; equal numbers of bobbins rotate in opposite directions, causing yarns to interlace into a braid pattern. The details of this pattern are defined by the number of bobbins used, spool pattern, mandrel diameter and the 'take-up speed' at which the braid is drawn forward [14].

Braiding over structured metal surfaces offers the possibility of incorporating advanced joining mechanisms at high production rates. Gude et al. [15] extensively reviewed contemporary manufacturing techniques for the fabrication of composite driveshafts with hybrid end-fittings, and presented several concepts of advanced driveshaft designs. These authors also compared mechanical properties obtained from different braid patterns and manufacturing processes. It was shown that the design of such parts requires not only consideration of different materials and braiding configurations, but also the geometry and positioning of surface features with respect to fibre yarns.

Optimisation of hybrid braided joints is a very complex task, since it involves maximising the load transfer between metal and fibres while also satisfying several manufacturing constraints related to the braiding process. Effective optimisation strategies will therefore require a deep understanding of the braiding process, since predicting and controlling the positioning of yarns over structured surfaces is key for both cost and performance. However, there currently are no modelling tools available to simulate the braiding process over complex structured mandrels accurately and efficiently.

The literature on braiding process modelling is limited; yet previous work can be broadly categorised into two types: geometrical-kinematic models, and those based on Finite Element (FE) analysis. Zhang et al. [16] presented a kinematic model of yarn motion within the 'convergent zone' in a circular braid, focusing on the effects of yarn straightness and frictional forces on final yarn distribution. Kessels and Akkerman [17] and Akkerman and Villa-Rodríguez [18] developed modelling approaches to predict the final fibre angle on complex arbitrary biaxial braided preforms, accompanied by experimental validation. In their work, yarns were assumed perfectly straight and free from interactions with other yarns, so that individual yarns could be analysed independently. Equations for idealised fibre paths at different stages within in the convergent zone were discretised and solved numerically. This approach was later applied to the optimisation of the braiding process [19].

Braiding simulations based on FE analysis offer several advantages over geometric-kinematic models, including consideration of complex geometries and nonlinear 
interactions between yarns. In this way, all factors influencing braid quality, such as friction between yarns, sliding contact against the guide ring, and frictional contact against the mandrel are considered as individual variables [20-22]. The studies however used single chains of beam or bar elements to represent each yarn, and were limited to smooth (i.e. non-structured) mandrels [20-22]. It should be noted that some complex phenomena observed in reality, such as yarn cross-sectional deformation and yarn splitting around surface protrusions, could not be fully captured. In addition, these analyses [20-22] appear to have been tailored to the manufacture of specific components, and as such would lack the versatility and scalability required for a more general use. For example, yarns had to be modelled in their entire lengths from the very beginning of the analysis, with several thousands of beam elements stretching out radially away from the guide ring, as if attached to an immense braiding wheel. This may result in great computational cost as well as the possibility of introducing kinematic errors. A fully scalable modelling technique would instead be preferred, especially when simulating long braids and/or multiple overbraids.

The approaches proposed in the literature do not seem suitable for virtual braiding trials over structured mandrels. Numerical modelling cannot be replaced by physical testing either, as it would have to consider an extremely large number of combinations between different braiding parameters and surface patterns. A modelling framework capable of conducting virtual braiding trials on structured mandrels is therefore in high demand. Moreover, if the model can predict detailed material characteristics such as fibre paths and final yarn cross-sections, then numerical models can be built for the virtual testing of final part performance.

In order to develop a better understanding of the braiding process over ALM surfaces, which is required to optimise the process, a systematic approach must be developed for detailed process simulations enabling 'virtual manufacture'. In this paper, a modelling framework is proposed and developed to simulate the process of braiding carbon fibres over structured mandrels, with enough accuracy and resolution to allow studies on the formation of common manufacturing defects, as well as being suitable for future analyses of final part performance.

Although the optimisation of hybrid metal-composite joints is the overarching objective, this article focuses on understanding and modelling the braiding process itself, in particular for mandrels containing detailed surface features. For this reason, the experimental work presented here was conducted using thermoplastic mandrels produced via ALM, instead of metallic ones, so that the modelling techniques could be verified and validated more easily and at a fraction of the cost. As will be discussed later, all modelling techniques described here can be directly applied to metallic mandrels, as long as the correct geometry, material properties, and surface properties are available.

The structure of the article is as follows. Section 2 describes the developed numerical framework including multiple options for the representation of fibre yarns; Sect. 3 presents the experimental setup used in the braiding of structured mandrels; Sect. 4 discusses the correlation between predicted and observed braid patterns; and finally Sect. 5 summarises the main findings, outcomes and areas of ongoing and future work.

\section{Braiding process modelling}

Figure 1 shows the schematic of a radial braiding machine, the type considered in the present work. Series of warp and weft spools move circumferentially in a counter-rotating fashion along the spool plane (the $x-y$ plane in Fig.1) with alternating $z$-axis translation to allow yarns to interlace. Three characteristic radii are defined, namely $R_{\mathrm{S}}, R_{\mathrm{G}}$ and $R_{\mathrm{M}}$ for the braiding wheel, guide ring and cylindrical mandrel, respectively.

In the present work the braiding process was modelled using the nonlinear explicit-dynamics solver LS-Dyna ${ }^{\circledR}$ version 9.0 [23]. All methods investigated here shared some common features, namely (1) all metal parts (i.e. cylindrical mandrel, surface protrusions and guide ring) were modelled as rigid bodies; (2) single or multiple strands of beam elements were used to represent individual yarns; and (3) general contact formulations were employed for inter-yarn interactions and contact against metal surfaces. Different strategies were adopted when modelling yarn feed and cross-sectional deformation, as discussed next.

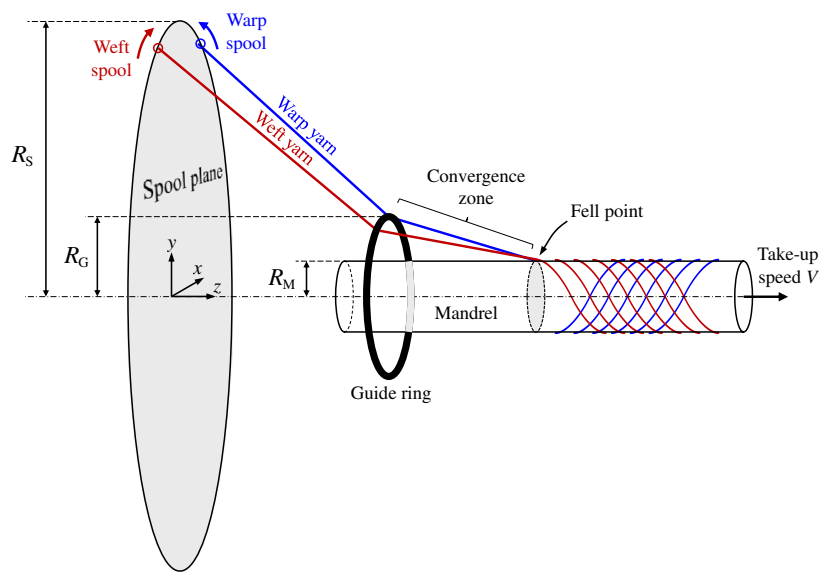

Fig. 1 Schematic of the braiding process 


\subsection{Finite single-strand (FSS) braiding model}

The finite single-strand (FSS) approach is the simplest of all braiding modelling techniques considered here. It is similar to the approaches presented in $[19,20]$, which appear to be the only contact-based simulation techniques reported in the open literature for braiding analysis. The FSS approach is therefore included here for completeness. It relies on the use of a single strand of beam elements to represent each yarn; multiple yarns are then modelled in their full lengths from the very beginning of the simulation. A FSS model is shown in Fig. 2. Strands of linear beam elements are used to represent yarns, while the guide ring and mandrel are modelled as rigid meshed surfaces.

A constant radial force is applied to each yarn to recreate the tension generated by spool and bobbin. Two components of motion are applied to each yarn ends which simulate the movement of carrier, as shown in Fig. 2. These components were (1) circumferential counter-rotating motion for weft and warp yarns, and (2) out-of-phase sinusoidal $z$-direction motion for interlacing. The mandrel was then driven along the $z$-axis at a constant take-up speed $V$.

Penalty-based contact pairs were defined for yarn-yarn, yarn-ring and yarn-mandrel interactions. Contact penalty stiffnesses were adjusted to avoid excessive interpenetration. In the absence of more accurate estimates, Coulomb friction coefficients for yarn-yarn and yarn-metal contacts were assumed to be 0.5 , based primarily on previous work reported in the literature [20]. Future work will focus on characterising these coefficients more accurately, e.g. via inverse modelling of experimental results. Automatic massscaling was employed to reduce the overall run time, defined by a target time step size (based on the Courant limit) of $1 \mu \mathrm{s}$. When employing the FSS method, all beam elements were given a circular cross-section, both for simplicity and consistency with previous work $[21,22]$.

Note from Fig. 2 that the effective wheel radius $R_{\mathrm{S}}$ decreases as braiding proceeds. This highlights one of the major disadvantages of the FSS method, i.e. it may result in unrealistic boundary conditions being applied to yarns.

\subsection{Infinite single-strand (ISS) braiding model}

In order to offer more realistic braiding boundary conditions, and to improve computational efficiency, it is better to 'feed' beam elements into the simulation as the braiding process evolves. This leads to the Infinite Single-Strand (ISS) yarn modelling approach. Numerical entities called 'beam sources' are used to represent individual bobbins as shown in Fig. 3. These entities allow for beam elements to be created on demand as the strand is pulled away from the source. New beam elements are created as and where necessary, which reduces simulation run time significantly. Input parameters defining each beam source include their spatial
Fig. 2 Braiding simulation using the finite single-strand (FSS) approach

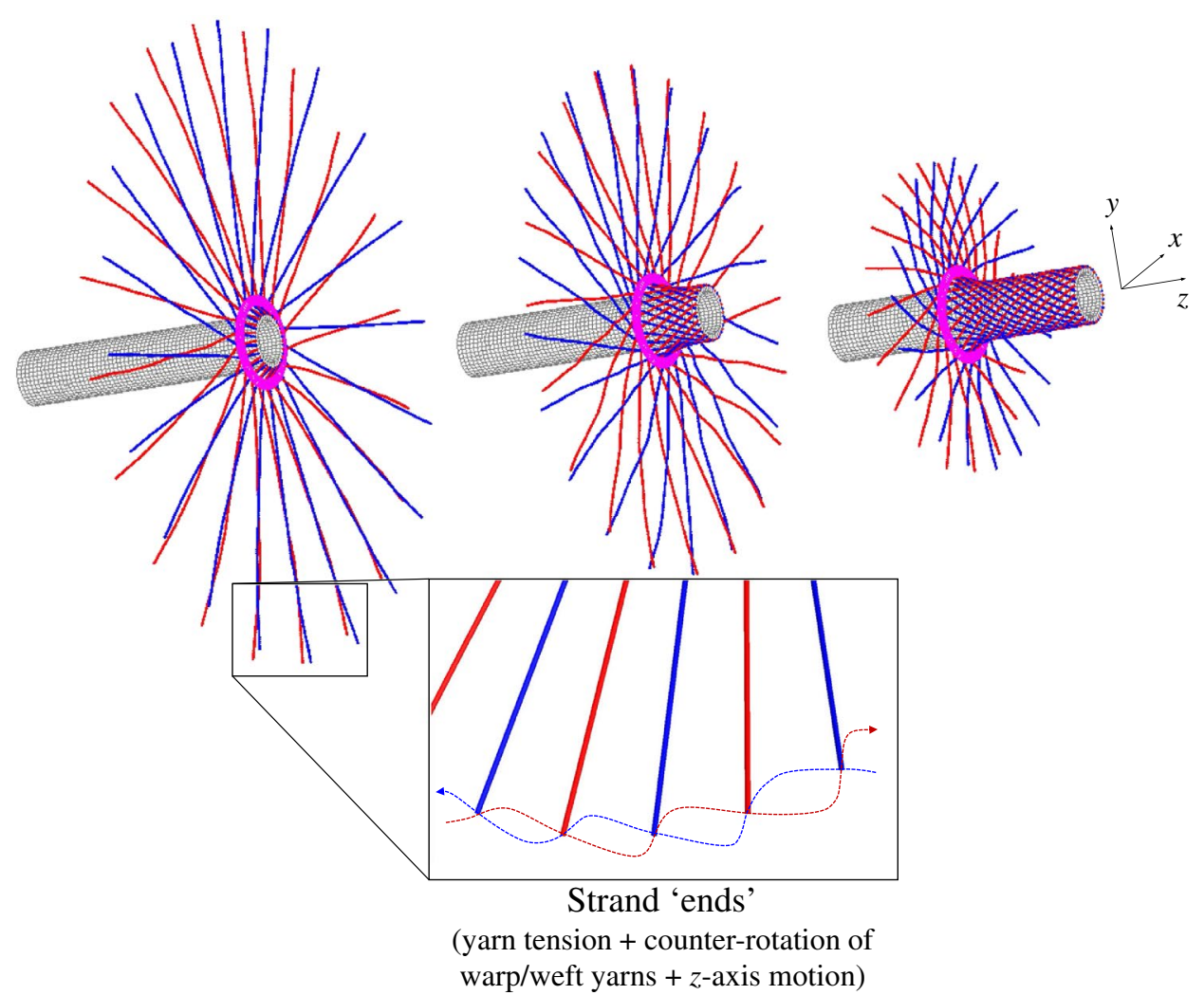


Fig. 3 Braiding simulation using the Infinite single-strand (ISS) approach
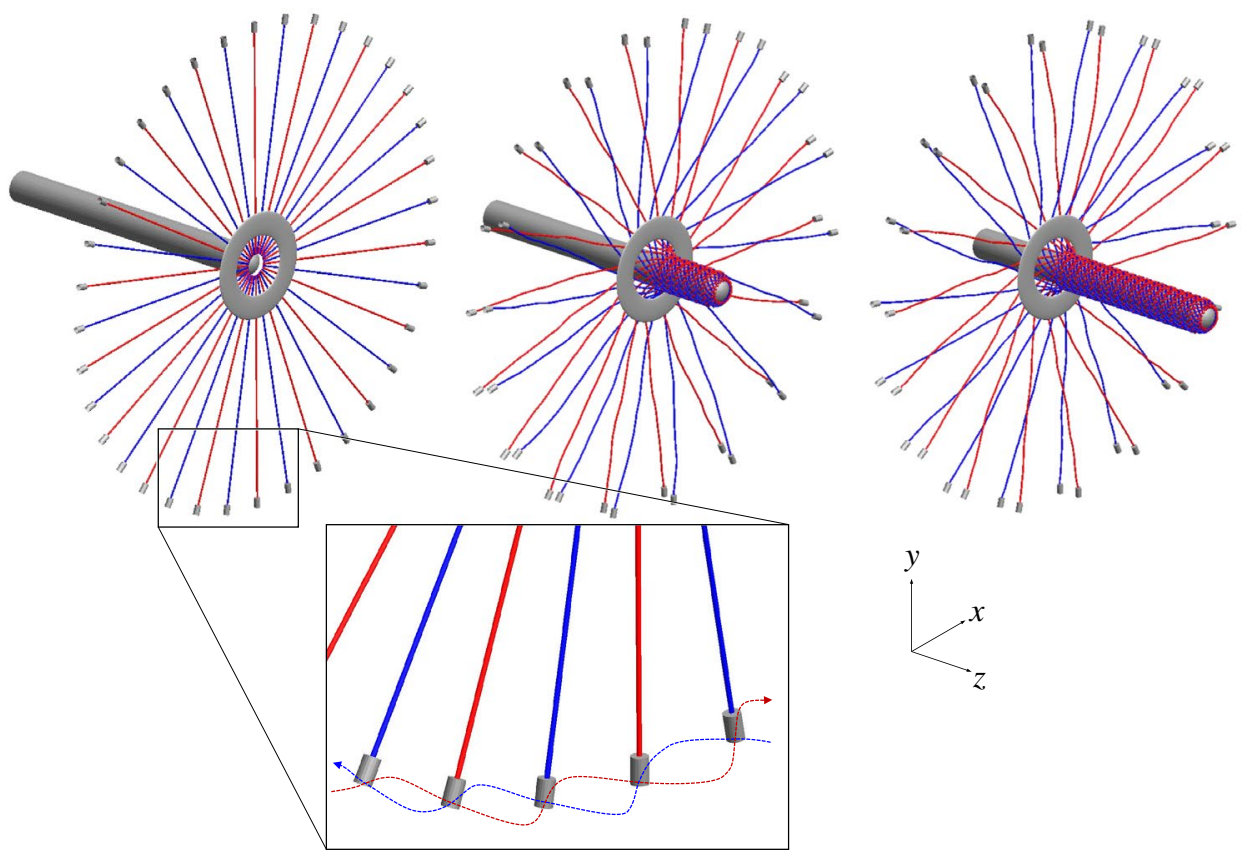

'Bobbins' (beam sources) (yarn tension + counter-rotation of warp/weft yarns $+z$-axis motion)

(Cartesian) coordinates and the tensional force 'resisting' strand extension.

Figure 3 shows that the motion of bobbins in ISS models follows a similar pattern as before, i.e. circumferential counter-rotation and out-of-phase $z$-axis translation. However, the effective wheel radius $R_{\mathrm{S}}$ is now correct and constant, resulting in more realistic yarn-yarn interactions throughout the braiding process.

All simulations were run on the Linux cluster 'Bluecrystal' at the University of Bristol, which at the time of writing had a total of 3568 compute cores running at $2.6 \mathrm{GHz}$. A typical $45^{\circ}$ braiding simulation over a mandrel length of $450 \mathrm{~mm}$ took typically $1 \mathrm{~h}$ of wall clock time twhen running on 16 cores.

\subsection{Tri-axial braids and overbraids}

The ISS approach is considerably more efficient than the FSS method and can therefore be used in more complex braiding scenarios. Figure 4 a shows an example of tri-axial braiding, i.e. including $0^{\circ}$ yarns, obtained by adding extra 'stationary' beam sources along the spool plane. Figure $4 \mathrm{~b}$ shows the result of an overbraid simulation obtained by running a second analysis step over the existing braid.

Two options have been tested when simulating overbraids. The first consisted of braiding over the previous model directly, by restarting the analysis with a take-up speed of $-V$. The second option, more accurate but also

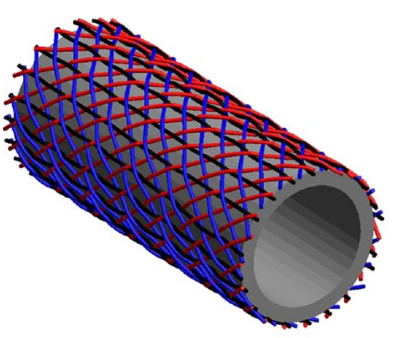

(a)

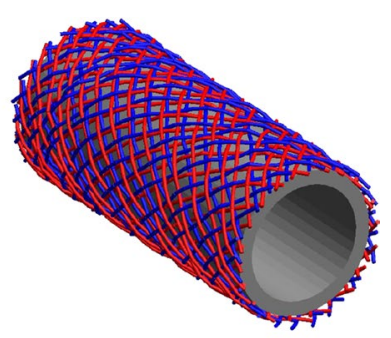

(b)
Fig. 4 a Tri-axial braiding simulation (i.e. including $0^{\circ}$ yarns) and $\mathbf{b}$ bi-axial overbraid simulation

costlier, was to apply mesh 'post-processing' techniques before each new overbraid was laid.

\subsection{Post-processing of single-strand braid models}

Models based on single strands of beam elements are mostly suitable for the estimation of final braiding angles over smooth mandrels with any cross section shapes. By assuming a constant yarn cross-section, these approaches cannot predict final yarn deformation and/or final fibre coverage accurately. In order to improve the prediction capability of single-strand yarn models, two 'post-processing' techniques were developed as shown in Fig. 5.

The assembled beam section (ABS) approach consisted of converting single beam elements into bundles of beams which together represent a single yarn. This approach is 


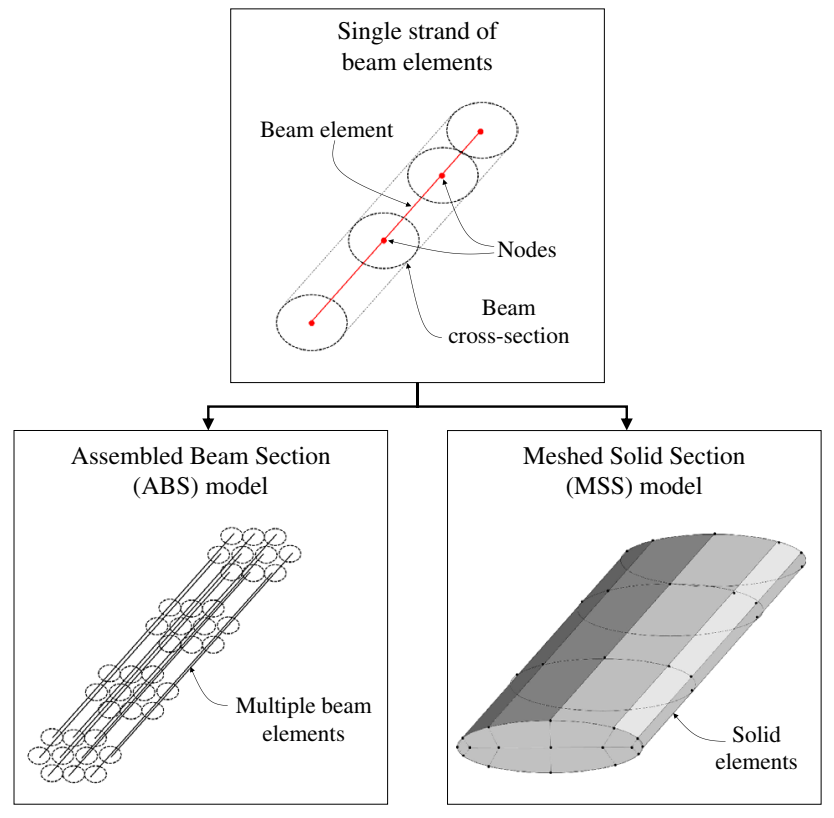

Fig. 5 Two post-processing strategies for 'single-strand' braid models

similar to the 'multi-chain digital element' technique proposed by Green et al. [24] for the analysis of 3D woven preforms. The ABS procedure was implemented here as a Matlab $^{\circledR}$ script which read LS-Dyna ${ }^{\circledR}$ model data and computed nodal coordinates for each new beam in the bundle. The number of beams to be added was defined by the user, and a good balance between geometrical accuracy and computational cost had to be found, leading to the baseline number of 12 beams per bundle. This is obviously far less than the number of fibres in real yarns, so each beam still represents thousands of fibres. The ABS algorithm estimated the yarn cross-sectional deformation based on yarn orientation with respect to the mandrel's surface. Assumptions regarding the average 'spread' of each yarn were controlled by user-defined input. In order to further improve deformation predictions, an optional 'dynamic relaxation' step could be introduced whereby further tension was applied to braided yarns until a final equilibrium configuration was reached. Figure $6 \mathrm{~b}$ shows an example ABS model obtained from the ISS braid in Fig. 6a.

The Meshed Solid Section (MSS) approach was also based on the work by Green et al. [24]. Yarn cross-sections were represented by meshes of three-dimensional 8-node solid elements, as shown at the bottom-right corner of Fig. 5. Typically 12 solid elements were used to represent a yearn cross-section, with element lengths (along yarns) equalling the original beam element length. As before, yarn crosssections were assumed to be elliptical, with dimensions controlled by user-defined input parameters. Likewise, a dynamic relaxation step could be added to produce more realistic braid geometries. Figure $6 \mathrm{c}$ shows an example MSS model obtained from the same ISS braiding simulation in Fig. 6a.

\subsection{Infinite multiple-strand (IMS) braiding model}

The post-processing steps described above made several assumptions regarding yarn deformation, and hence required the definition of several input parameters. Furthermore, single-strand yarn models cannot capture yarn splitting which is a common manufacturing defect. To address these shortcomings the Infinite Multiple-Strand (IMS) model
Fig. 6 Post-processing of $\mathbf{a}$ an ISS braiding model into b ABS and $\mathbf{c}$ MSS models
Infinite Single-Strand (ISS) braiding model
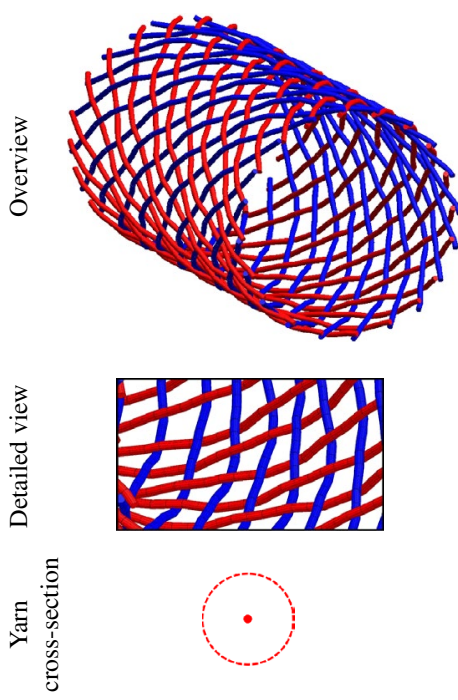

(a)
Assembled Beam Section (ABS) model
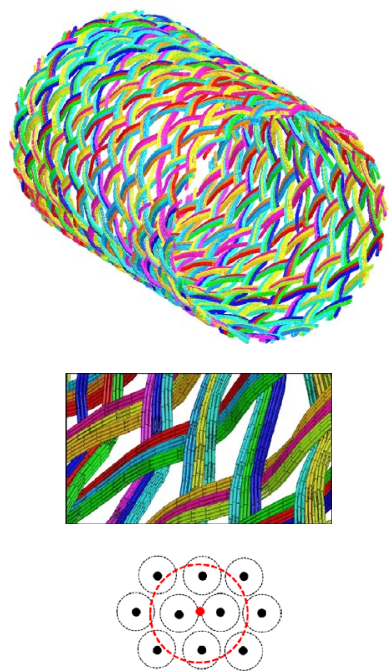

(b)
Meshed Solid Section (MSS) model
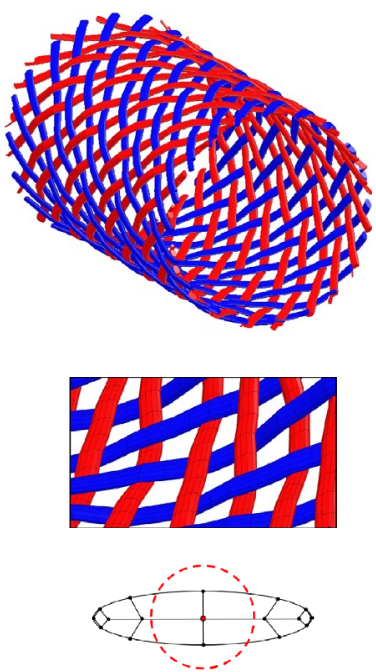

(c) 
was developed, whereby multiple strands of beam elements were used to represent each yarn over the entire braiding simulation.

Every new strand of beams added to the model increases computational cost in proportion to the number of yarns in the braid. In order to minimise computational overhead, parametric studies were conducted by varying the number of strands per yarn. Although more strands resulted in more realistic deformed cross-sections, it was found that three strands per yarn enabled satisfactory predictions of manufacturing defects while still offering acceptable run times. Experimental observations have shown that yarns rarely undergo 'symmetric' splitting; instead they tend to split near one of the 'edges', at least for the pin configurations investigated here. This observation further supports the use of three strands per yarn. An example of the IMS modelling approach is shown in Fig. 7.

In the IMS model, beam radii and separation distance between strands can be adjusted to match the real yarn width. Moreover, the central strand in a three-strand yarn model may be given a slightly larger radius to better represent an elliptical cross-section. However, for braiding simulations involving structured mandrels, it was found that a constant radius across all strands gave better predictions of yarn splitting. Figure 8 shows typical yarn splitting and twisting predictions using the fixed-radius three-strand IMS approach.
When compared with experimental data, IMS models were found to predict well the density of manufacturing defects.

\section{Braiding experiments}

\subsection{Specimen design}

As discussed in Sect. 1, developing a suitable braiding process model is a pre-requisite for the optimisation of hybrid metal-composite shafts. Since the braiding process itself results in relatively small loads being applied to the mandrel, thermoplastic pinned sleeves produced by ALM were used in this work, since their wide availability and low cost meant that several braiding trials could be conducted quickly during the early stages of model development. The use of thermoplastic inserts also facilitates characterisation via X-ray computed tomography $(\mathrm{CT})$, which will be useful when verifying internal layers in overbraids.

These surrogate end-fittings were fabricated by fused deposition of Acrylonitrile Butadiene Styrene thermoplastic. The process included a sacrificial 'support material' which was chemically removed after the ALM process was complete. Typical fittings used in this work can be seen in Fig. 9a.
Fig. 7 Braiding simulation using the infinite multiplestrand (IMS) approach
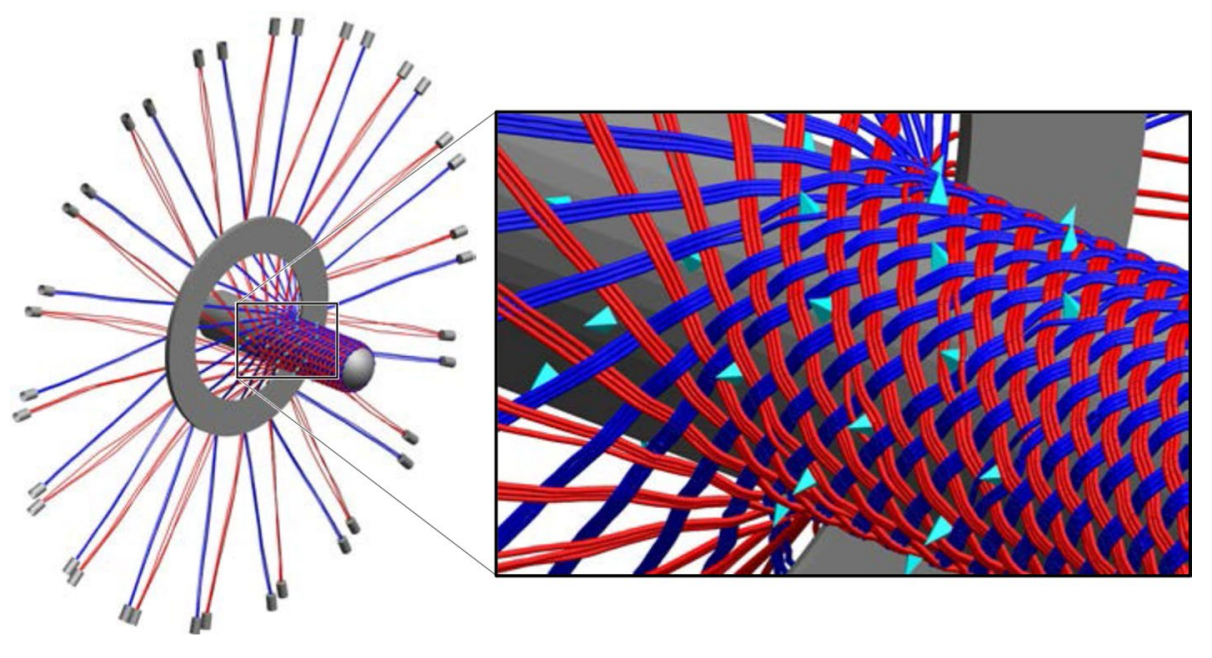

Fig. 8 Manufacturing defects captured by an IMS model; a yarn splitting, b splitting and twisting

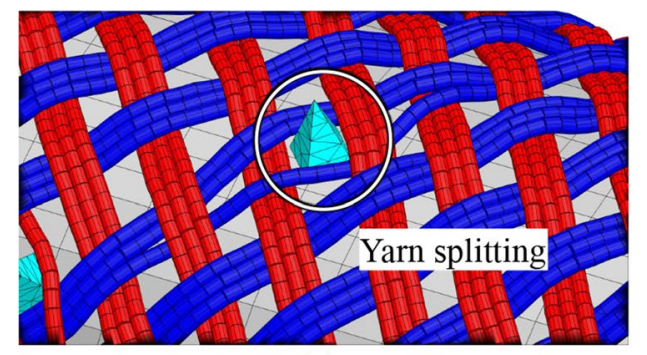

(a)

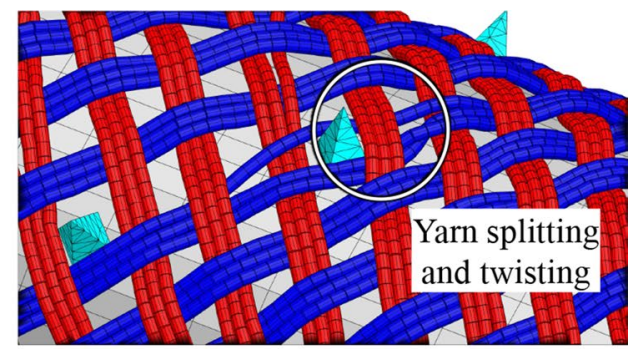

(b) 
Fig. 9 a Thermoplastic pinned sleeves and $\mathbf{b}$ mandrel assembly including sleeves, spacers and end cap

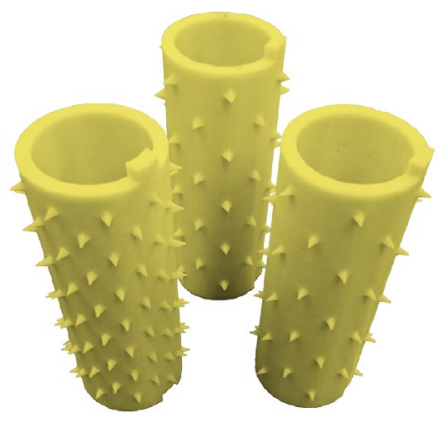

(a)

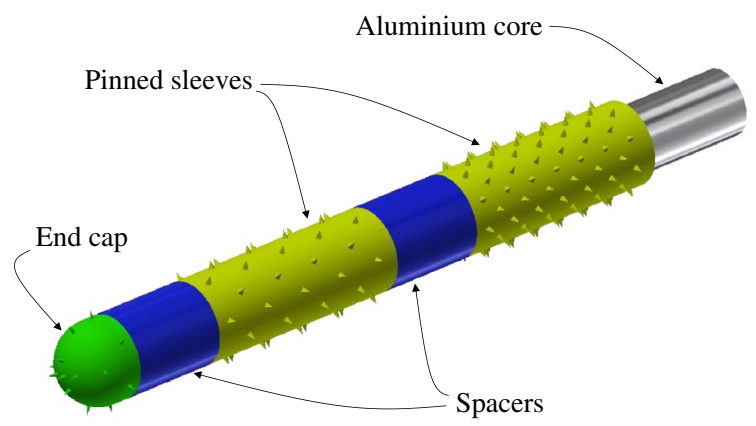

(b)

Fig. 10 Dimensions of pinned sleeves; a pin height $h$ and pin width $a, \mathbf{b}$ baseline sleeve with 54 pins, and $\mathbf{c}$ high pin density sleeve with 117 pins

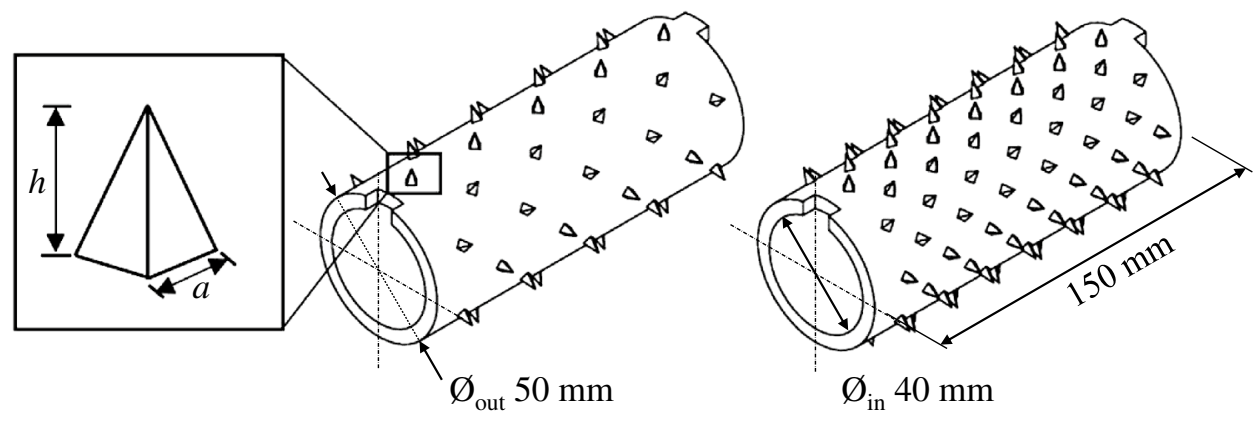

(a)

(b)

(c)

Multiple ALM 'sleeves' were then fitted on to a rigid aluminium alloy tube as shown in Fig. 9b, with plastic spacers inserted in between sleeves and an end cap added to the tip of the tube. Apart from the aluminium tube, all other components were fabricated via ALM. The end cap also contained small pins to facilitate initial alignment and to prevent slippage during the initial stages of braiding. Single and multiple overbraid trials were conducted. After each test, the fibre preform was 'stabilised' over the mandrel by tying plastic cable ties at the two ends of the mandrel assembly.

Different pin arrangements were considered in this preliminary study, with all pins being pyramidal and rotated by $45^{\circ}$ with respect to the braiding axis. The geometry of pins was defined by two parameters, namely the height $h$ and width $a$ as shown in Fig. 10a.

The performance of hybrid joints is believed to be strongly influenced by the shape of surface protrusions, as well as their areal density and spatial arrangement. Higher protrusion densities are expected to promote a more direct load transfer between metal and fibres, as well as greater reinforcement in the through-thickness direction. However, protrusion geometry and arrangement also influence the braiding process, especially the formation of manufacturing defects (e.g. yarn splitting, bridging and twisting) and the final braid thickness.
Table 1 Pinned sleeve configurations

\begin{tabular}{llllr}
\hline $\begin{array}{l}\text { Sleeve } \\
\text { design }\end{array}$ & $\begin{array}{l}\text { Pin height } \\
(\mathrm{mm})\end{array}$ & $\begin{array}{l}\text { Pin width } \\
(\mathrm{mm})\end{array}$ & $\begin{array}{l}\text { Sleeve } \\
\text { length }(\mathrm{mm})\end{array}$ & Number of pins \\
\hline 1 & 4 & 2 & 150 & 54 \\
2 & 4 & 2 & 150 & 117 \\
3 & 4 & 4 & 150 & 54 \\
4 & 4 & 4 & 150 & 117 \\
\hline
\end{tabular}

In this work four different pin configurations were considered as shown in Table 1 . The pin height $h$ was kept constant at $4 \mathrm{~mm}$, and two pin widths were tested, namely 2 and $4 \mathrm{~mm}$. Pin arrangements were based on integer multiples of the average spacing between adjacent weft and wrap yarns for a $50 \mathrm{~mm}$ diameter unpinned mandrel. Two pin densities were then considered, resulting in a 'baseline' $150 \mathrm{~mm}$ long sleeve containing 54 pins (Fig. 10b) and a high pin-density sleeve with 117 pins (Fig. 10c).

\subsection{Braiding equipment and data acquisition}

Braiding tests were carried out at the Institute of Lightweight Engineering and Polymer Technology (ILK) at TU Dresden. Their setup, shown in Fig. 11a, consisted of a Herzog $^{\circledR}$ RF1/288-100, 4.7 m diameter braiding machine 
Fig. 11 a Braiding setup at TU Dresden, and $\mathbf{b}$ camera positions around the braiding wheel (camera 2 displaced temporarily to reveal the guide ring)

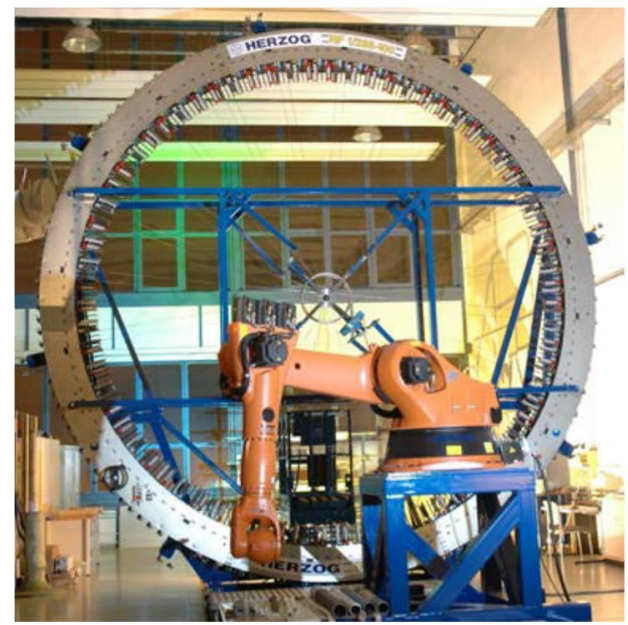

(a)

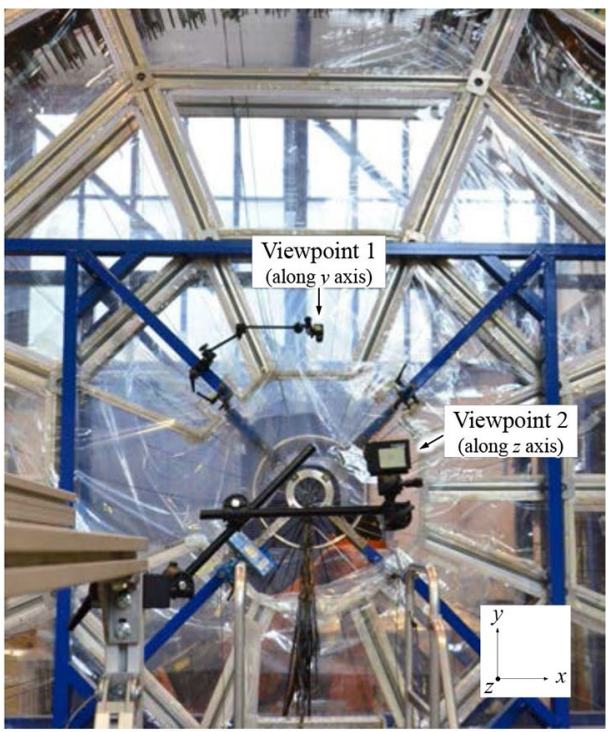

(b)

\section{Results and discussion}

Table 2 Specification of the braiding setup

\begin{tabular}{ll}
\hline Property & Value \\
\hline Yarn properties & $\begin{array}{c}\text { Toray } \\
\text { fibre } \text { T700 }\end{array}$ \\
& $24 \mathrm{~K}$ \\
Braider inner diameter, $2 R_{S}$ & $4.7 \mathrm{~m}$ \\
Braiding style & $1 \mathrm{by} 1 \mathrm{bi}^{-a x i a l}$ \\
Take-up speed, $V$ & $1.8 \mathrm{~mm} \mathrm{~s}^{-1}$ \\
Mandrel radius, $R_{M}$ & $50 \mathrm{~mm}$ \\
Rotational speed of carriers, $\omega$ & $0.0727 \mathrm{rad} \mathrm{s}^{-1}$ \\
Number of yarns & 36 \\
\hline
\end{tabular}

in combination with a $\mathrm{KUKA}^{\circledR} \mathrm{KR} 210 \mathrm{~L} 180-2$ robotic arm. The details of the setup are listed in Table 2 .

In order to capture the braiding process from different angles, two C-mount ' $4 \mathrm{k}$ ' digital cameras $(3840 \times 2160$ pixels@30 fps)with telephoto lenses were positioned at different points around the braiding wheel as shown in Fig. 11b. The camera at viewpoint 1 was placed directly above the braiding exit to capture the braiding convergence zone. The camera at viewpoint 2 was aligned with the mandrel (i.e. along the $z$-axis) to capture a projection of the braiding process on to the $x-y$ plane. Braiding trials on both pinned and unpinned mandrels were conducted, and analyses of pin arrangement and braid quality were made. Images taken during the braiding process were then used for model verification and validation.

\subsection{Unpinned mandrels}

The different modelling strategies discussed in previous sections were first verified against experiments using mandrel assemblies similar to that shown in Fig. 9b, but with unpinned thermoplastic sleeves. FE models were built according to the experimental setup listed in Table 2, and several virtual braiding trials were conducted.

The 'nominal' braiding angle $\alpha$ is set by controlling the ratio between the angular velocity of carriers and the take-up speed. The relationship is [14],

$\alpha=\arctan \frac{\omega R_{M}}{V}$,

where $\omega, R_{M}$ and $V$ are the rotational speed of carriers, mandrel radius and take-up speed, respectively. However, when analysing model results it is possible to extract 'local' braiding angles for each beam element in the model. Figure 12 shows such data for three different nominal braid angles, namely $30^{\circ}, 45^{\circ}$ and $60^{\circ}$, as predicted by the ISS model on unpinned mandrels (for clarity, data from only one weft and one warp yarn are shown). The apparent 'oscillation' in local braid angles is due to the interlacing of warp and weft beam elements as they go over and under each other. This effect is more noticeable for larger braid angles, as the interlacing 'density' increases. Local braid angles vary widely in the very beginning of the process, as yarns interact with the hemispherical end cap, but quickly converge towards the nominal value. It should be noted that lower nominal braid angles $\left(30^{\circ}\right.$ in this case) require the most braid length before 


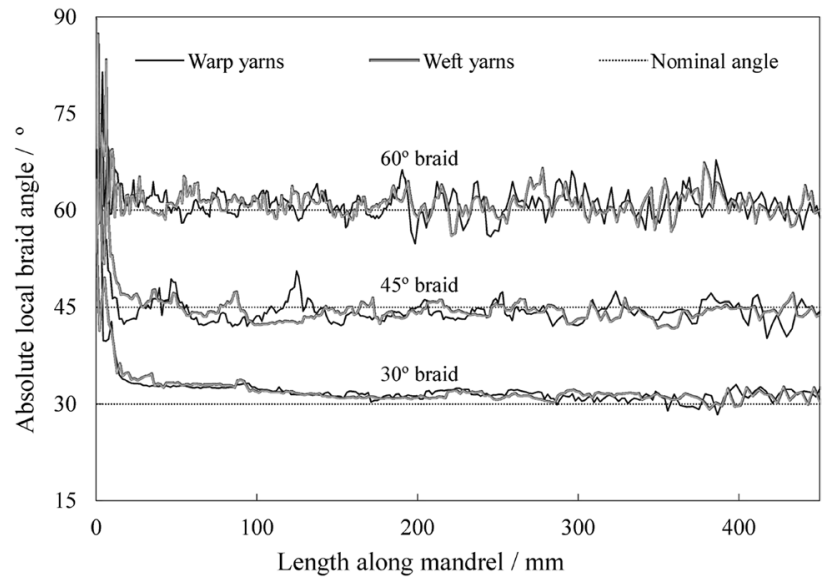

Fig. 12 Local braid angles vs. length along mandrel as predicted by the ISS model

full convergence (around $200 \mathrm{~mm}$ for the $30^{\circ}$ case) while larger angles show almost immediate convergence.

Figure 13 shows experimental and virtual braids obtained using ISS and IMS models. Very good agreement is observed in terms of final braid patterns and braid angles. It should be noted however that the single-strand modelling approach results in a slight over-prediction of braid thickness and under-prediction of braid coverage, as expected since the single cylindrical beam sections are not accurate representations of real braided yarns. On the other hand, the multiple-strand model resulted in considerably more realistic braid coverage and thickness, as expected, but with simulation run times approximately 2.6 times higher than those using the single-strand approach.

Imagery of braiding 'convergence zones' were also analysed and compared. Figure $14 \mathrm{a}$ shows a typical steady-state convergence zone as seen from viewpoint 1 , which is then compared with the IMS model prediction in Fig. 14b. Predicted convergence zone lengths were in good agreement with observations; however, considerable variations in yarn spacing were observed in experiments, which can be attributed to local variations in yarn width (affecting local

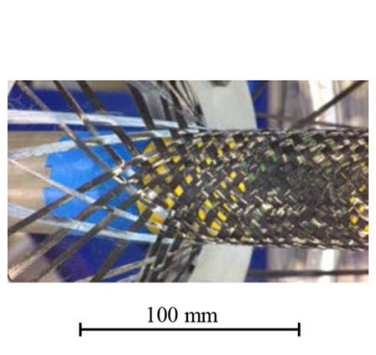

(a)

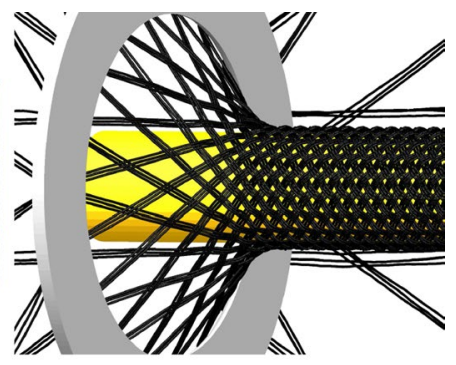

(b)
Fig. 14 Braiding convergence zone as seen from viewpoint 1; a experiment, b IMS model (both figures share the same scale)

frictional properties), as well as yarn twisting and splitting within the braiding wheel. The FE model predicts a much more regular yarn spacing, since yarn cross-sections are virtually constant, and no twisting or splitting are observed before the convergence zone is reached.

Figure $15 \mathrm{a}$ and $\mathrm{b}$ show observed and predicted yarn spacings, respectively, as seen from viewpoint 2. Again, very good qualitative agreement is obtained, with a more regular interlacing pattern being predicted by the FE model in comparison to real observations, which may be again attributed to local variations in yarn geometry and frictional properties.

\subsection{Pinned mandrels}

Studies on the virtual braiding of pinned mandrels focused primarily on verifying model prediction capabilities in terms of the formation of braiding defects, such as yarn splitting and bridging. Figure 16 compares the quality of single braids over sleeves of low and high pin densities. Both cases show considerable numbers of braid imperfections around pins, predominantly yarn bridging and splitting.

Evidence of the model's prediction capabilities was shown in Fig. 8, which depicts numerical results for the lower pin density. The IMS model provided good qualitative agreement with experimental observations; full quantitative agreement can only be checked once parameters such as
Fig. 13 Observed yarn coverage; a experiment, $\mathbf{b}$ ISS model, and $\mathbf{c}$ IMS model. The 'braid angle' is defined as shown in $\mathbf{b}$

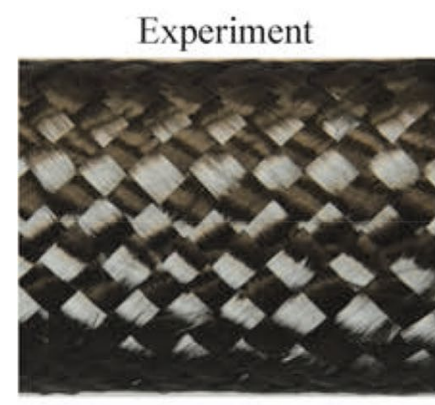

(a)

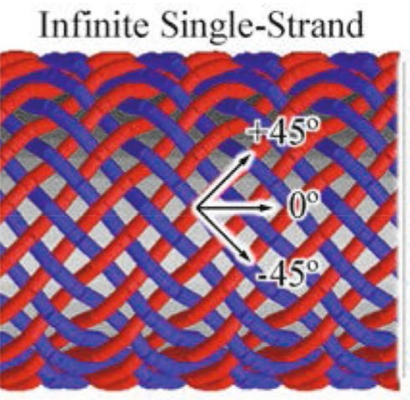

$50 \mathrm{~mm}$

(b)

\section{Infinite Multiple-Strand}

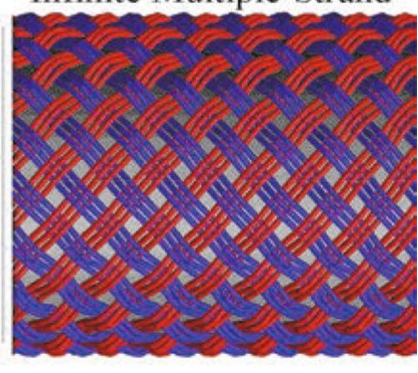

(c) 
Fig. 15 Braiding convergence zone as seen from viewpoint 2 ; a experiment, b IMS model (both figures share the same scale)

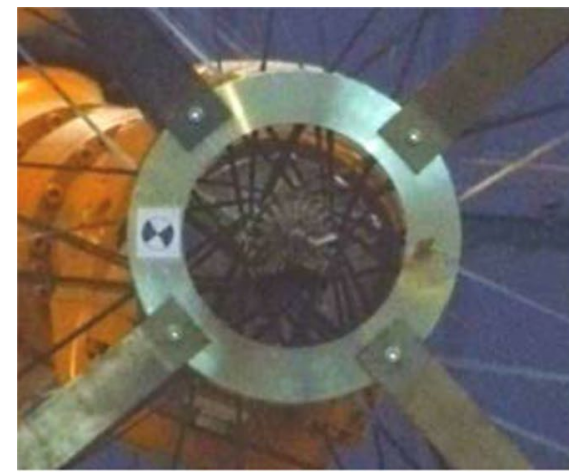

(a)

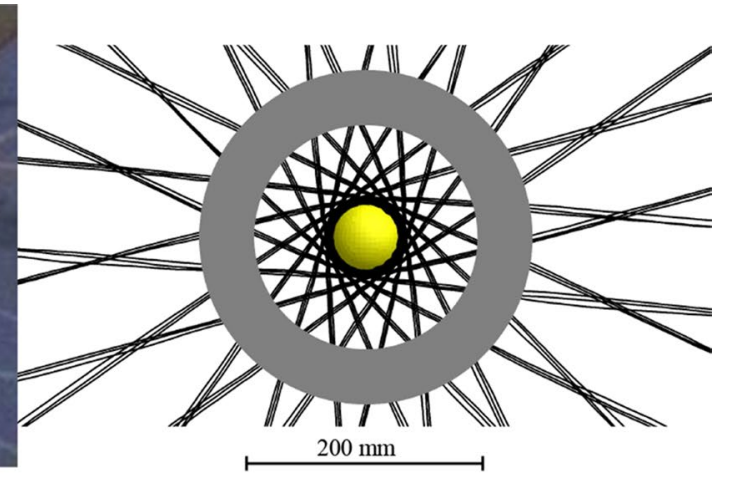

(b)

Fig. 16 Quality of braid over pinned mandrels; a low pin density and $\mathbf{b}$ high pin density

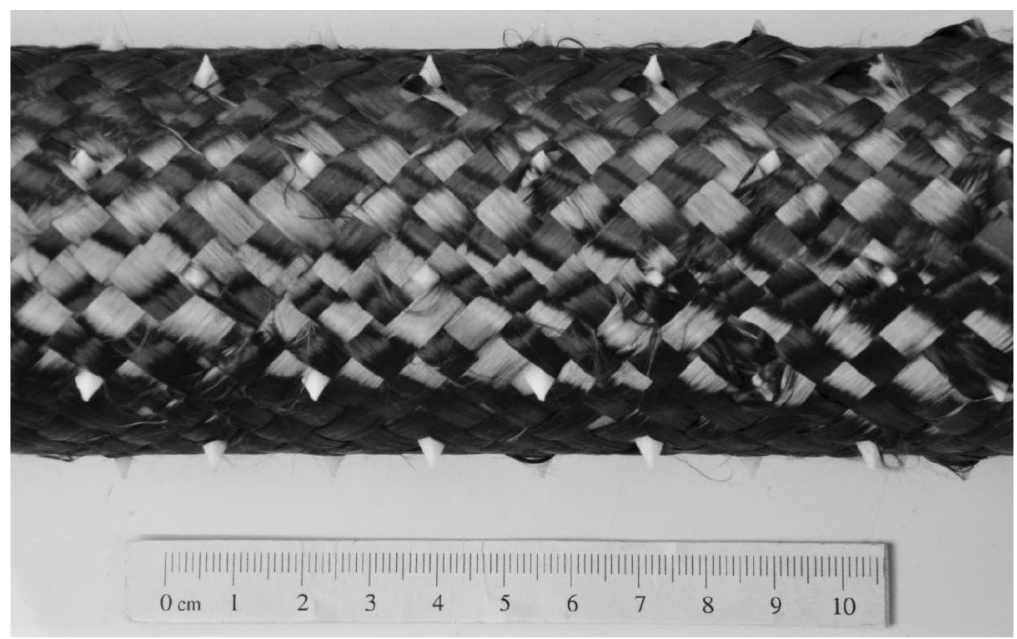

(a)

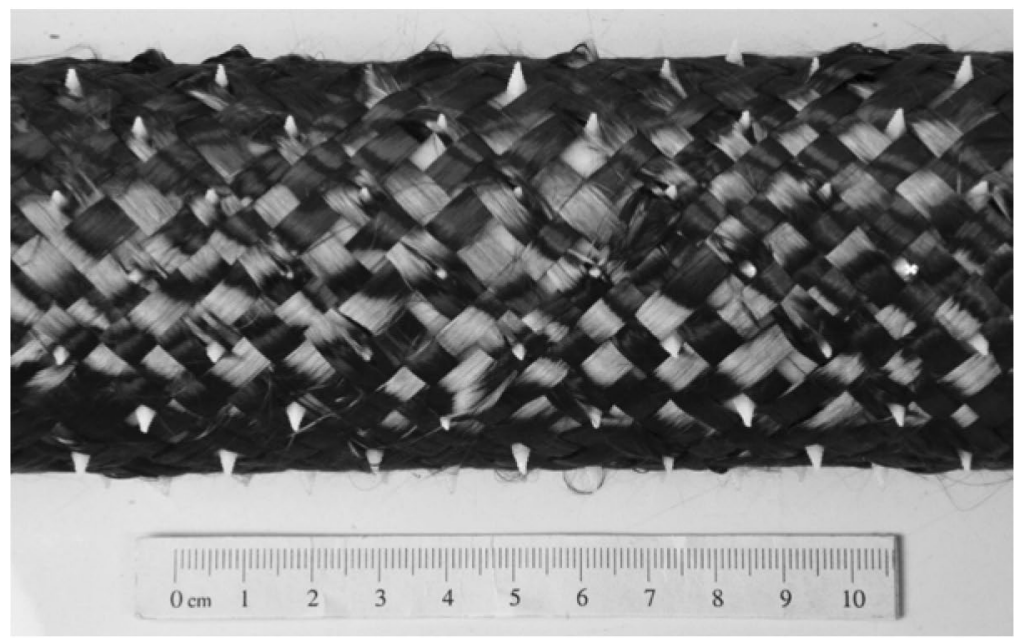

(b)

coefficients of friction have been fully identified. When the baseline friction coefficient of 0.5 was used, yarn splitting became the most common manufacturing defect; in order to reproduce the prevalence of yarn bridging seen in experiments, the coefficient of friction between yarns and pins had to be increased to approximately 0.7 . This suggests that the surface roughness created by ALM processes contributes to the occurrence of bridging defects, and that artificially high friction coefficients may be required in FE models to compensate for their perfectly smooth surfaces.

Figure 17 shows the fibre paths predicted by the IMS model, using baseline friction coefficients, for low and high 
Fig. 17 Fibre paths predicted by the IMS model; a low pin density and $\mathbf{b}$ high pin density

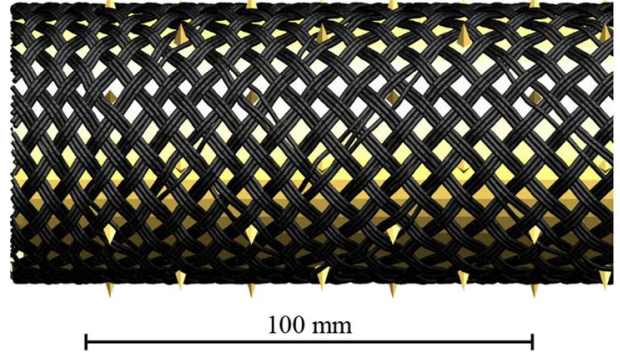

(a)

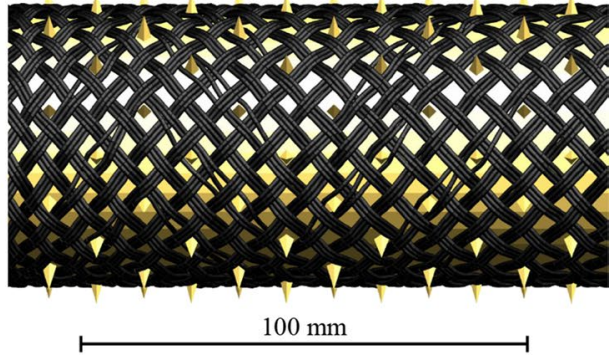

(b) pin densities. Yarn splitting can be seen in both cases, similar to the experimental observations in Fig. 16. Once initiated, yarn splits tended to propagate over a number of pins in a row, following the braid angle $\left(45^{\circ}\right.$ in this case). Pins disturb the braid pattern and cause variations in local braid angle. This results in small but detectable peaks/troughs in plots of local braid angle, as shown in Fig. 18 for low and high pin densities. The relatively small number of such peaks and troughs, despite the large number of braid imperfections observed, confirms the tendency of yarn splits to span several pins in a row.

In order to gauge the accuracy of the model when predicting the occurrence of braiding defects, the number of pin-led imperfections was counted over each braided sleeve, in experiments and models. Figure 19 compares the percentages of pins causing braid imperfections for both pin densities (because each pinned sleeve in this study had a unique combination of pin geometry and arrangement, the data in Fig. 19 relate to individual configurations and therefore no measures of variability are available). Some discrepancy is observed between model predictions and experimental observations, but both sets of results suggest a lower percentage of defects for the high pin density case.

Possible reasons for the differences between models and experiments include minor variations in local geometry, material properties, contact properties, and test setup. In order to investigate the latter, a parametric study was conducted on the effects of introducing an initial misalignment angle $\theta$ between the pin pattern and the oncoming yarns. Although the sleeves were carefully aligned with respect to the braiding wheel at the start of each experiment, the actual value of $\theta$ in real life is unknown. In the FE models, this misalignment angle was introduced by turning the mandrel slightly about its axis prior to the start of the simulation. Because 36 yarns were used, the braid pattern is expected repeat itself every $10^{\circ}$ about the mandrel axis. Therefore, the misalignment angle was varied within the range $0 \leq \theta<10^{\circ}$, in increments of $2.5^{\circ}$, plus a single negative value of $-5^{\circ}$ for completion. The resulting percentages of pin defects for the 117-pin sleeve are shown in Figure 20. The number of observed braiding (a) Unpinned

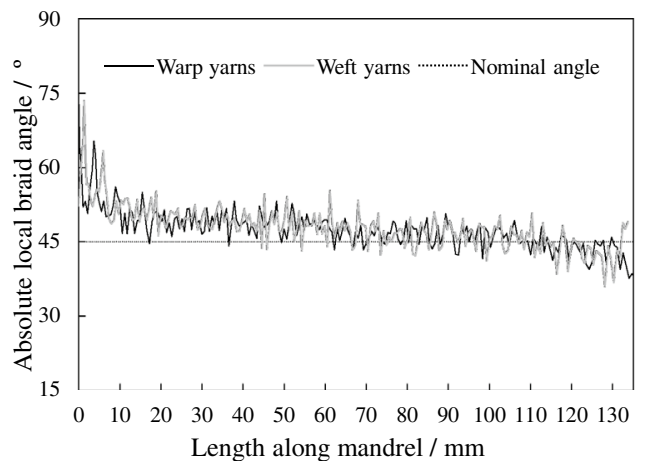

(b) 54 -pin
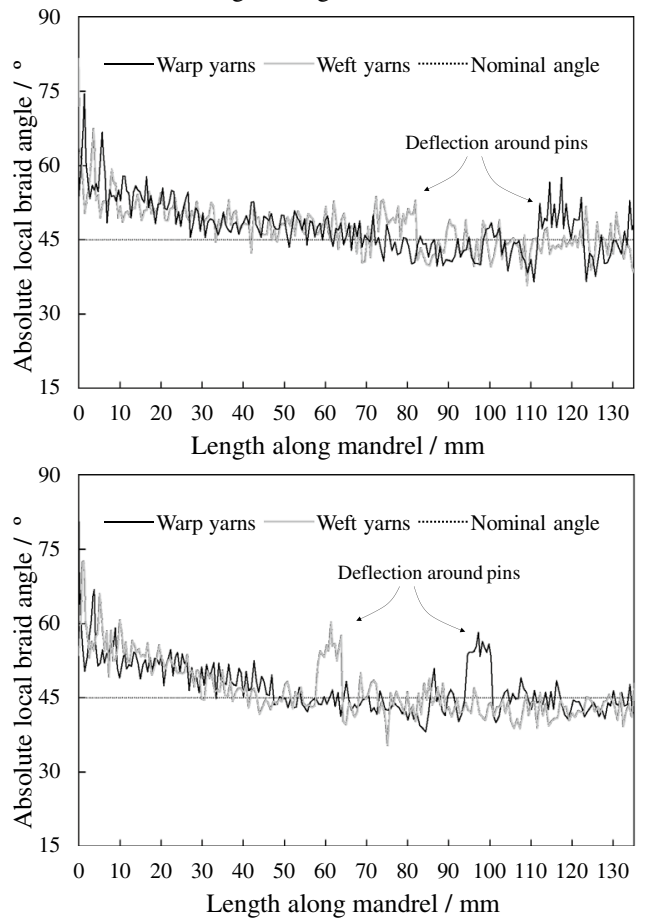

Fig. 18 Local braid angle, as predicted by the IMS model, for a unpinned sleeve, b 54-pin sleeve and c 117-pin sleeve

imperfections appears to be strongly affected by this initial misalignment, being lowest for the IMS model when $\theta=5^{\circ}$. This suggests that the initial alignment in the model was not optimal, and that the discrepancy between 


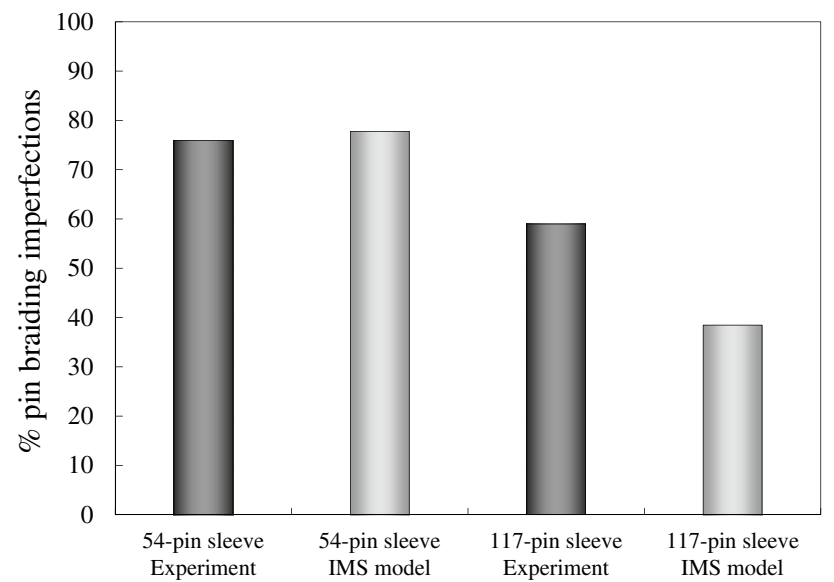

Fig. 19 Percentage of pin braiding imperfections as observed experimentally and predicted by simulations

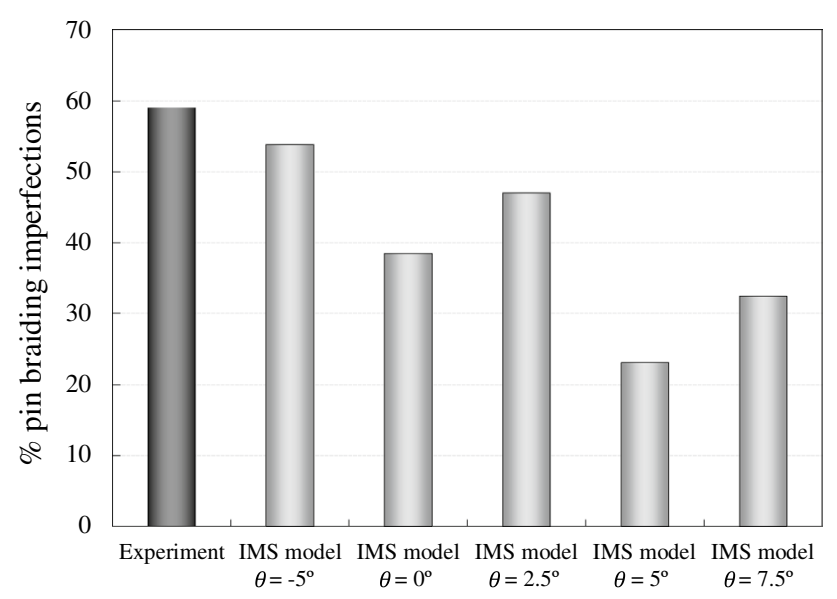

Fig. 20 Effect of mandrel misalignment angle $\theta$ on the percentage of pin braiding imperfections for the 117-pin sleeve, as predicted by the IMS model

predictions and observations in Fig. 19 could, at least in part, be attributed to variations in the test setup.

Overall, this study confirmed the inevitability of braiding defects being introduced when pin-like protrusions are present. This is because yarns undergo large numbers of contact interactions during the braiding process, including extensive frictional sliding within the convergence zone, which cause random variations in yarn positioning with respect to pins. Consequently, defect-free braids could not be obtained even is simulations. However, the models presented here can be used in the future in a more rigorous statistical characterisation of braid quality and sensitivity to unknown variables.

\section{Conclusions}

Series of experimental and numerical studies were carried out to investigate the braiding of metal-fibre hybrid joints for the manufacture of high-performance hybrid driveshafts. Nonlinear finite element models of full braiding/overbraiding processes were created using single or multiple strands of beam elements for each yarn, and accounting for yarn deformation and frictional contact. The infinite multiplestrand (IMS) modelling approach-with three strands of beam elements per yarn-was found to offer the best balance between accuracy and computational cost. This was the simplest and fastest model capable of reproducing yarn splitting and yarn bridging, the two most common manufacturing defects when pinned mandrels are involved.

Braiding trials were conducted using thermoplastic sleeves produced via additive layer manufacture (ALM) as surrogates for metallic parts. The numerical models were verified successfully against experiments on the prediction of final braid angle and occurrence of manufacturing defects. Full model validation will require the identification, through independent experiments, of a number of unknowns (e.g. friction coefficients). However, the proposed modelling framework was shown to be numerically stable, robust and scalable, and therefore suitable for the "virtual manufacturing' of candidate designs for braided hybrid joints.

The models presented here are equally suitable for the analysis of braided joints made of other material combinations, and work is currently ongoing on the simulation and physical testing of metallic end-fittings produced by ALM.

Acknowledgements XC. Sun and L.F. Kawashita acknowledge funding by the UK Engineering and Physical Sciences Research Council (EPSRC) grant number EP/M021963/1. The authors would like to thank Steffen Zillmann at TU Dresden for conducting the braiding trials, assisted by the University of Bristol students Robert Peterson, Robbie E. Herring and Charles R.J. Ebdon.

Open Access This article is distributed under the terms of the Creative Commons Attribution 4.0 International License (http://creativeco mmons.org/licenses/by/4.0/), which permits unrestricted use, distribution, and reproduction in any medium, provided you give appropriate credit to the original author(s) and the source, provide a link to the Creative Commons license, and indicate if changes were made.

\section{References}

1. Cartié DD, Cox BN, Fleck NA (2004) Mechanisms of crack bridging by composite and metallic rods. Compos Part A Appl Sci Manuf 35(11):1325-1336. https://doi.org/10.1016/j.compo sitesa.2004.03.006

2. Ucsnik S, Scheerer M, Zaremba S, Pahr D (2010) Experimental investigation of a novel hybrid metalcomposite joining technology. Compos Part A Appl Sci Manuf 41(3):369-374. https://doi. 
org/10.1016/j.compositesa.2009.11.003. http://linkinghub.elsev ier.com/retrieve/pii/S1359835X09003728

3. Stelzer S, Ucsnik S, Pinter G (2016) Strength and damage tolerance of compositecomposite joints with steel and titanium through the thickness reinforcements. Compos Part A Appl Sci Manuf 88:39-47. https://doi.org/10.1016/j.composites a.2016.05.020. http://linkinghub.elsevier.com/retrieve/pii/ S1359835X16301415

4. Graham D, Rezai A, Baker D, Smith PA, Watts JF (2011), A hybrid joining scheme for high strength multi-material joints. In: 18th International Conference on Composite Materials (ICCM), 21-26 August 2011, Jeju, South Korea, pp 1-6

5. Wang X, Ahn J, Kaboglu C, Yu L, Blackman BRK (2016) Characterisation of composite-titanium alloy hybrid joints using digital image correlation. Compos Struct 140:702-711. https://doi. org/10.1016/j.compstruct.2015.12.023

6. Tu W, Wen P, Guild F (2010) The optimization of Comeld (TM) Joints: a novel technique for bonding composites and metal. In: Lecture Notes in Engineering and Computer.. II (June) 2-4. URL: URL: http://www.iaeng.org/publication/WCE2010/WCE2010_ pp1383-1385.pdf

7. Kashaev N, Ventzke V, Riekehr S, Dorn F, Horstmann M (2015) Assessment of alternative joining techniques for Ti-6Al-4V/CFRP hybrid joints regarding tensile and fatigue strength. Mater Design 81:73-81. https://doi.org/10.1016/j.matdes.2015.04.051

8. Ko MG, Kweon JH, Choi JH (2014) Fatigue characteristics of jagged pin-reinforced composite single-lap joints in hygrothermal environments. Compos Struct 119:59-66. https://doi. org/10.1016/j.compstruct.2014.08.025

9. Nguyen ATT, Brandt M, Orifici AC, Feih S (2016) Hierarchical surface features for improved bonding and fracture toughness of metal-metal and metal-composite bonded joints. Int J Adhes Adhes 66:81-92. https://doi.org/10.1016/j.ijadhadh.2015.12.005

10. Nguyen AT, Brandt M, Feih S, Orifici AC (2016) Pin pull-out behaviour for hybrid metal-composite joints with integrated reinforcements. Compos Struct 155:160-172. https://doi. org/10.1016/j.compstruct.2016.07.047 http://linkinghub.elsev ier.com/retrieve/pii/S0263822316312648

11. Nguyen AT, Amarasinghe CK, Brandt M, Feih S, Orifici AC (2017) Loading, support and geometry effects for pin-reinforced hybrid metal-composite joints. Compos Part A Appl Sci Manuf 98:192-206. https://doi.org/10.1016/j.compositesa.2017.03.019

12. Parkes PN, Butler R, Almond D (2013) Fatigue of metal-composite joints with penetrative reinforcement, 54th AIAA/ASME/ ASCE/AHS/ASC Structures, Structural Dynamics, and Materials Conference, Structures, Structural Dynamics, and Materials and Co-located Conferences. https://doi.org/10.2514/6.2013-1879
13. Parkes PN, Butler R, Meyer J, De Oliveira A (2014) Static strength of metal-composite joints with penetrative reinforcement. Compos Struct 118:250-256

14. Kyosev Y (2014) Braiding Technology for Textiles - Principles, Design and Processes. 1st edn.Woodhead Publishing, Cambridge, UK

15. Gude M, Lenz F, Gruhl A, Witschel B, Ulbricht A, Hufenbach W (2015) Design and automated manufacturing of profiled composite driveshafts. Sci Eng Compos Mater 22(2):187-197. https://doi. org/10.1515/secm-2014-0048

16. Zhang Q, Beale D, Broughton R (1999) Analysis of circular braiding process, Part 1: theoretical investigation of kinematics of the circular braiding process. J Manuf Sci Eng 121(3):345-350. https ://doi.org/10.1115/1.2832687

17. Kessels J, Akkerman R (2002) Prediction of the yarn trajectories on complex braided preforms. Compos Part A Appl Sci Manuf 33(8):1073-1081. https://doi.org/10.1016/S1359-835X(02)00075 -1. http://linkinghub.elsevier.com/retrieve/pii/S1359835X020007 51

18. Akkerman R, Villa-Rodríguez BH (2006) Braiding simulation for RTM preforms. In: 8th International conference on Textile Composties (Texcomp 8), 16-18 October 2006, Nottingham, UK, vol 1081, p 7500

19. van Ravenhorst J, Akkerman R (2013) Optimisation of the circular braiding process. In: TexComp 11 Conference, 19-20 September 2013, Leuven, Belgium. https://doi.org/10.1006/mssp.1996.0136. http://doc.utwente.nl/88474/1/TexComp11AkkermanandRaven horstBraiding.pdf

20. Pickett AK, Sirtautas J, Erber A (2009) Braiding simulation and prediction of mechanical properties. Appl Compos Mater 16(6):345-364. https://doi.org/10.1007/s10443-009-9102-x

21. Hans T, Cichosz J, Brand M, Hinterhölzl R (2015) Finite element simulation of the braiding process for arbitrary mandrel shapes. Compos Part A Appl Sci Manuf 77:124-132. https://doi. org/10.1016/j.compositesa.2015.06.003. http://www.sciencedir ect.com/science/article/pii/S1359835X1500202X

22. Swery EE, Hans T, Bultez M, Wijaya W, Kelly P, Hinterhölzl R, Bickerton S (2017) Complete simulation process chain for the manufacturing of braided composite parts. Compos Part A Appl Sci Manuf 102:378-390. https://doi.org/10.1016/j.composites a.2017.08.011

23. Livermore Software Technology Corporation (LSTC), URL: http://www.lstc.com/products/ls-dyna LS-Dyna (2017). URL: http://www.lstc.com/products/ls-dyna

24. Green SD, Long AC, El Said BSF, Hallett SR (2014) Numerical modelling of 3D woven preform deformations. Compos Struct 108:747-756. https://doi.org/10.1016/j.compstruct.2013.10.015 